\title{
Live imaging of collagen deposition during experimental hepatic schistosomiasis and recovery: a view on a dynamic process
}

\author{
Marina C. G. Harvie ${ }^{1} \cdot$ Donald P. McManus ${ }^{1} \cdot$ Hong You $^{1} \cdot$ Vanessa A. Rivera ${ }^{1} \cdot$ Sujeevi K. Nawaratna ${ }^{1}$. \\ Kelli P. A. MacDonald ${ }^{1}$. Grant A. Ramm ${ }^{1,2}$ - Geoffrey N. Gobert $\mathbb{D}^{1,3}$
}

Received: 15 June 2018 / Revised: 26 July 2018 / Accepted: 26 July 2018 / Published online: 6 November 2018

(c) United States \& Canadian Academy of Pathology 2018

\begin{abstract}
Hepatic fibrosis is the central cause of chronic clinical pathology resulting from infection by the blood flukes Schistosoma japonicum or $S$. mansoni. Much has been elucidated regarding the molecular, cellular and immunological responses that correspond to the formation of the granulomatous response to trapped schistosome eggs. A central feature of this Th2 response is the deposition of collagen around the periphery of the granuloma. To date, traditional histology and transcriptional methods have been used to quantify the deposition of collagen and to monitor the formation of the hepatic granuloma during experimental animal models of schistosomiasis. We have investigated the dynamic nature of granuloma formation through the use of a transgenic mouse model (B6.Collagen 1(A) luciferase mice (B6.Coll 1A-luc ${ }^{+}$)). With this model and whole-animal bioluminescence imaging, we followed the deposition of collagen during an active schistosome infection with Chinese and Philippines geographical strains of S. japonicum and after clearance of the adult parasites by the drug praziquantel. Individual mice were re-imaged over the time course to provide robust real-time quantitation of the development of chronic fibrotic disease. This model provides an improved method to follow the course of hepatic schistosomiasis-induced hepatic pathology and effectively supports the current dogma of the formation of hepatic fibrosis, originally elucidated from static traditional histology. This study demonstrates the first use of the B6.Coll 1A-luc ${ }^{+}$mouse to monitor the dynamics of disease development and the treatment of pathogen-induced infection with the underlying pathology of fibrosis.
\end{abstract}

\section{Introduction}

Schistosomiasis is a chronic disease caused by trematode Schistosoma spp parasites. It is a condition affecting at least 200 million people globally and is the second most prevalent non-bacterial infection after malaria [1]. In

Electronic supplementary material The online version of this article (https://doi.org/10.1038/s41374-018-0154-0) contains supplementary material, which is available to authorized users.

Geoffrey N. Gobert

g.gobert@qub.ac.uk

1 QIMR Berghofer Medical Research Institute, Brisbane, QLD, Australia

2 Faculty of Medicine, The University of Queensland, Brisbane, QLD, Australia

3 School of Biological Sciences, Queen's University Belfast, Belfast, UK
Asia, schistosomiasis japonica remains a significant public health issue, particularly in China and the Philippines. Several distinct geographical strains of Schistosoma japonicum have been reported including those identified from Taiwan, Indonesia, Japan, China and the Philippines [2]. The Chinese ( $\mathrm{SjC}$ ) and Philippines ( $\mathrm{SjP})$ forms of $S$. japonicum exhibit a number of discrete morphological and other phenotypic differences, including different pre-patent periods, tegument topography, adult worm size, virulence and the sub-species of snail utilised as intermediate host $[2,3]$.

Schistosomes have a complex lifecycle involving both a mammalian definitive and an aquatic snail intermediate host. The mammalian host is penetrated by cercariae released into freshwater from infected intermediate snail hosts in endemic areas. The cercariae lose their tails and mature to the juvenile schistosomula stage as they traverse the blood vascular system of the mammalian host, eventually arriving in the lungs. After a brief period in the lungs, the schistosomula continue to mature and migrate, 
ultimately residing in the hepatic portal vein as a pair of mature male and female worms in copula. The eggs laid by female worms are responsible for the pathology associated with schistosomiasis [4]. Many eggs travel through the intestinal wall and are passed in the faeces, hatching externally in fresh water to release miracidia, which infect the intermediate host snails. However, owing to the blood flow in the portal vein, some eggs are swept into the liver where they instigate the development of fibrotic granulomas. It is this hepatic fibrosis that causes the chronic pathology characteristic of schistosomiasis [5].

The use of animal models and traditional histology to investigate and observe the development of hepatic fibrosis requires the terminal sacrifice of the animals to process and analyse infected tissues, thus permitting only cross-sectional point-in-time observations. Here we have applied a dynamic imaging model to investigate the realtime development of fibrosis and hepatic schistosomiasis in individual live mice over time. This disease platform is a valuable tool that can both reduce the number of mice required for each experiment and provide robust dynamic quantitative data of collagen deposition. With the capacity to follow the temporal development of fibrosis in an animal host, curative drug treatment may be administered at the appropriate time, which may more accurately reflect the clinical setting. Recovery from disease or fibrosis regression may also be followed in individual animals. This unique model represents a powerful tool to study the effects of various treatment regimens on the schistosome-induced burden of hepatic disease.

The model uses a luciferase-based transgenic reporter mouse (B6.Coll1A-luc+) that expresses luciferin under the control of the pro $\alpha 1(\mathrm{I})$ collagen (COL1A1) promoter [6]. When collagen is translated, the enzyme luciferase is co-expressed, which acts to cleave any luciferin substrate present, generating a bioluminescent signal that can be detected using an appropriate in vivo imaging system. As the luciferase gene is expressed concurrently with collagen, the dynamic deposition of newly transcribed collagen, but not existing collagen deposits, can be quantified. B6.Coll1A-luc + mice have been used to study collagen deposition in chemically induced liver fibrosis in a hepatotoxic injury model [7]. Since the pathology of schistosomiasis japonica or mansoni infection is dominated by liver fibrosis [8, 9], we have utilised this mouse model to provide valuable information on the realtime development of collagen deposition and recovery. This work represents a new model for studying fibrosis and the resultant pathology associated with hepatic schistosomiasis.

\section{Materials and methods}

\section{Mice}

B6.collagen 1(A) luciferase mice (B6.Coll 1A-luc+) were used as a heterozygous cross on a C57B16/J background and were supplied by the QIMR Berghofer Medical Research Institute Animal Facility (Brisbane, QLD, Australia). These mice have the procollagen $\alpha 1(\mathrm{I})$ promoter transgene linked to a firefly luciferase gene [6]. Mice were housed in micro-isolator cages and received normal chow as feed. Mice were age and sex matched, and, when their weight was $>20 \mathrm{~g}$, were infected by abdomen exposure with $S$. japonicum cercariae. For $\mathrm{SjP}$ (S. japonicum Philippine-Sorsogon strain) female mice were used; while for SjC ( $S$. japonicum Chinese (Anhui) strain) both male and female mice were used.

\section{Bioluminescence imaging}

Imaging was performed using a Xenogen (Xenogen IVIS 100, Caliper Life Sciences, Hopkinton, MA) in vivo imaging system, using standard settings. Mice were injected with $0.5 \mathrm{mg}$ of luciferin (Sigma-Aldrich), intraperitoneally, and then anaesthetised with isofluorane $5 \mathrm{~min}$ prior to imaging. Animals were imaged at weekly intervals as indicated. Data were analysed with the Living Image Software Version 4 (Caliper Life Sciences, Hopkinton, MA) and presented as photons per second per square centimetre per steer radiant.

\section{Schistosome infection}

Age- and sex-matched mice were infected percutaneously with either $\mathrm{SjP}$ (35 cercariae per mouse) or $\mathrm{SjC}$ (14 cercariae per mouse). Mice recovered in a warmed room $\left(28^{\circ} \mathrm{C}\right)$ before being returned to standard housing.

\section{Praziquantel (PZQ) treatment}

Between weeks 6 and 7 post-cercarial infection, a cohort of mice received PZQ (Sigma, USA) treatment to clear the S. japonicum worms. PZQ was dissolved in $2.5 \%(\mathrm{v} / \mathrm{v})$ cremophor EL (Sigma, USA) and was given by oral gavage as an escalating dose from 150 to $350 \mathrm{mg} / \mathrm{kg}$ over 5 days (total dose $1250 \mathrm{mg} / \mathrm{kg}$ ) [8]. The successful clearance of adult parasites in individual mice was confirmed during the imaging weeks by the cessation of faecal egg production, using standard parasitological methods [10]. The clearance of adult parasites was confirmed following perfusion of individual mice at the point of sacrifice. 

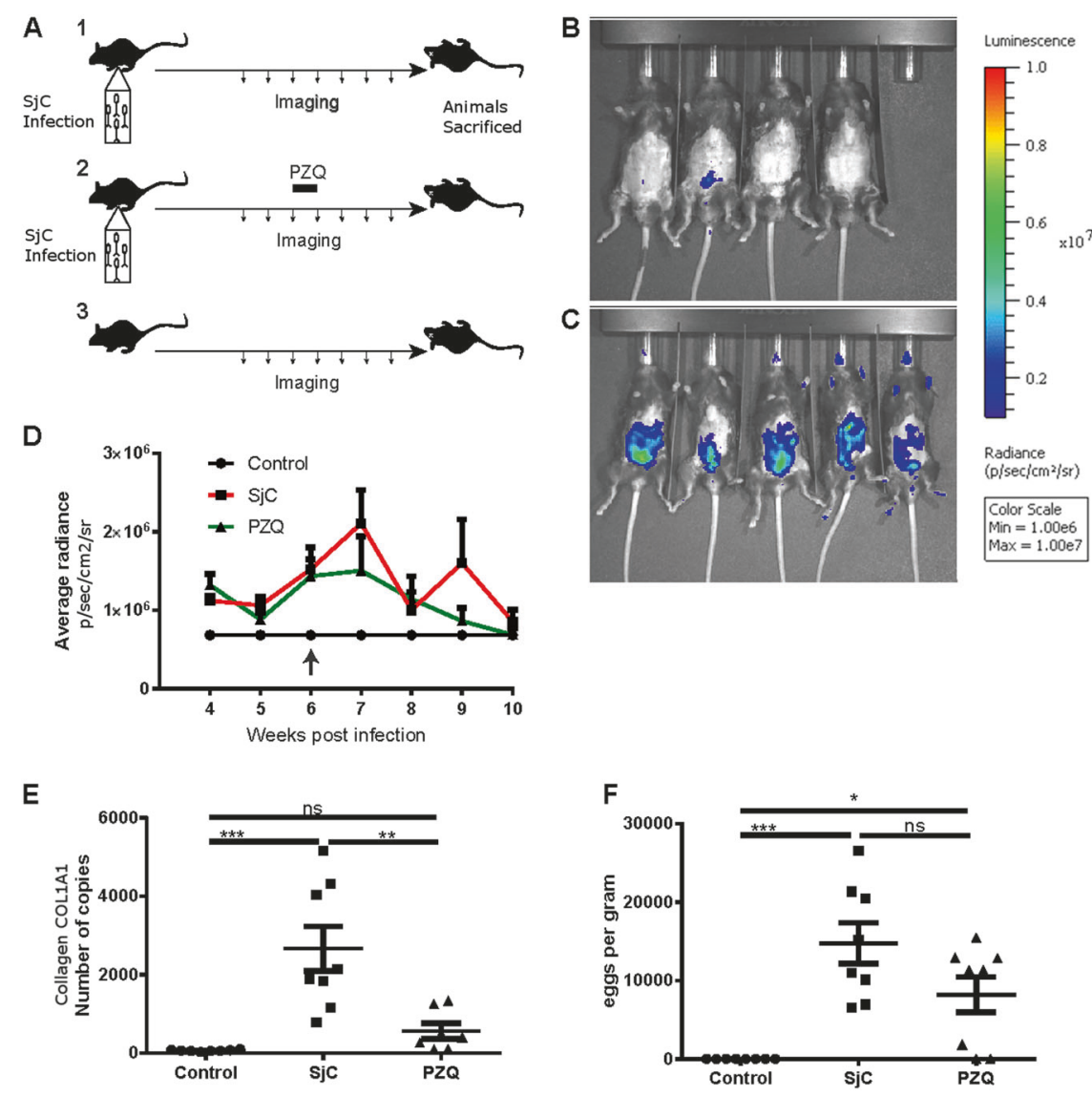

Fig. 1 Collagen deposition during $\mathrm{SjC}$ infection measured by IVIS dynamic imaging. Experimental overview (a): Male and female mice were separated into three cohorts: Group 1 was infected with $14 \mathrm{SjC}$ cercariae and imaged weekly from weeks 4 to 10; Group 2 was infected with $14 \mathrm{SjC}$ cercariae, imaged weekly from weeks 4 to 10 and treated with PZQ between weeks 6 and 7 to clear infection; Group 3 was an uninfected control group, imaged weekly from weeks 4 to 10 of the experiment. Mice were sacrificed after week 10. Representative images of control (b) and SjC-infected mice (c) undergoing IVIS imaging. Radiance scale (range) in $\mathrm{p} / \mathrm{s} / \mathrm{cm}^{2} / \mathrm{sr}$. d Average radiance of procollagen $\alpha 2$ (I) luciferase bioluminescence measured weekly over

\section{Experimental grouping}

Mice were organised into three experimental groups in order to compare bioluminescent imaging with traditional histology-based measures of fibrosis after schistosome infection (see Fig. 1a for an overview).

- Group 1 were infected with schistosome cercariae and disease was allowed to proceed (referred to as "SjP" or "SjC" in graphs depending on the schistosome strain used for the infection). For $\mathrm{SjC}$ infection, mice were imaged weekly from weeks 4 to 10 , post-cercarial infection. For $\mathrm{SjP}$ infection, mice were imaged weekly from weeks 6 to 11, post-cercarial infection. Mice from groups 2 and 3 were also imaged at these time points.

the infection time course $(n=10)$. Arrow represents onset of PZQ treatment. Statistics calculated using two-way ANOVA with Tukey post-test: Week $6\left({ }^{*}\right.$ Control vs $\mathrm{SjC}$, *Control vs PZQ), Week 7 (****Control vs $\mathrm{SjC}, *$ Control vs PZQ) Week $9(*$ Control vs $\mathrm{SjC})$. At all time points, SjC vs PZQ NS. e Real-time PCR of collagen gene expression in mice from the infected, PZQ-treated and control groups. f Enumeration of eggs deposited in the liver during $\mathrm{SjC}$ infection. Data are presented as means \pm SEM. Statistics calculated using two-way (d) or one-way ANOVA $(\mathbf{e}, \mathbf{f})$ with Tukey post-test. $* p \leq 0.05, * * p \leq 0.01$, $* * * p \leq 0.001, * * * * p \leq 0.0001$. NS $>0.05$

- Group 2 were infected and then at week 6 the infection was cleared by oral PZQ dosages (referred to as "PZQ" in graphs) after liver fibrosis and egg laying had occurred.

- Group 3 mice were uninfected and acted as controls and provided baseline levels of normal fibrosis within the liver (referred to as "control" in graphs).

Infections ("SjC/SjP" and "PZQ" groups) were allowed to progress until parasite egg laying was expected to begin (week 4-6 post-cercarial challenge) and then weekly whole-animal imaging was performed. Control mice were also imaged at each week and used as a baseline for subsequent analysis. After week 6 of infection, a subset of mice ("PZQ" group) were treated 
with the anthelmintic PZQ to clear the infection and allow for subsequent recovery of the liver tissue fibrosis due to parasite clearance and liver repair [8]. In the experiments with $\mathrm{SjC}$ parasites, mice were culled at the final time point of 10 weeks (or earlier on ethical grounds). SjPinfected mice were culled at various points during the experimental time course. In the $\mathrm{SjP}$ group, two mice were culled at week 8 and three mice were culled at week 10a corresponding three mice from the PZQ-treated group were also culled at week 10 .

\section{Liver egg counts}

Hepatic egg burdens were calculated as eggs per gram (EPG) of liver as described [11]. Briefly, eggs were extracted from a portion of liver of known mass by overnight digestion in $4 \%$ potassium hydroxide. Eggs were then resuspended in $1 \mathrm{~mL}$ of formalin and the number of eggs in three $5 \mu \mathrm{L}$ aliquots were counted and averaged to calculate the EPG.

\section{Histology}

Murine livers were harvested, preserved in $10 \%(\mathrm{v} / \mathrm{v})$ buffered formalin, embedded in paraffin and sectioned on microscope slides. Haematoxylin and eosin (H\&E) and Picro-Sirius Red single staining was performed as described [9]. Stained slides underwent virtual microscopy (ScanScope XT Slide Scanner, Aperio, Vista, USA) and were analysed with the ImageScope software version 11.2 (Aperio). Sirius Red-stained collagen fibres were quantified by the ImageScope software (analysis algorithm 'Positive Pixel v9', with Hue value: 0, Hue width: 0.1 and colour saturation threshold 0.05). Liver sections were selected digitally and blood vessels were excluded from the analysis.

\section{Real-time PCR}

Total RNA was extracted from liver tissue using Trizol (Invitrogen, Carlsbad, USA) and an RNeasy Mini Kit (Qiagen Inc., Hilden, Germany) [12]. All samples were treated with DNase (Promega, Madison, USA), and cDNA synthesised using a Quantitect Reverse Transcription Kit (Qiagen Inc). cDNA concentration was measured using a Nanodrop-1000 (Nanodrop Technologies, Wilmington, USA). Hypoxanthinephosphoribosyltransferase (HPRT) was used as a reference gene to normalise data from all experiments [11]. Real-time PCR was performed using SYBR Green master mix (Applied Biosystems, Foster City, USA) on a Corbett Rotor Gene 6000 (Corbett Life Sciences, Uithoorn, The Netherlands). Primer sequences used for COL1A1 and HPRT were as previously reported
$[8,9]$. Data were analysed by importing the standard curve to each run using the Rotor-Gene 6000 Series software (version 1.7).

\section{Statistical analysis}

Graphic illustration and statistical analysis was performed with GraphPad Prism version 7.02. Data were statistically analysed with one-way analysis of variance (ANOVA) or two-way ANOVA, with Tukey's multiple comparison, where indicated.

\section{Results}

\section{SjC-infected Coll Luc + mice}

\section{Collagen deposition visualised using IVIS imaging and quantitative PCR (qPCR) analysis}

The overview of this experiment is presented in Fig. 1a. The livers of uninfected control mice (Fig. 1b) were used to compare the deposition of collagen in $\mathrm{SjC}$ infected mice (Fig. 1c) after injection with luciferin and the detection of bioluminescence. Average radiance was measured in a defined abdominal region for individual mice for each time point and each treatment/ infection group. Over the course of infection, we were able to detect differences between infected, recovering and uninfected mice in reflected average radiance as determined by IVIS imaging. Between weeks 6 and 7 in the cohort of infected mice receiving PZQ to treat the worm infection and over time, we observed a decrease in average radiance in these animals after luciferin injection and IVIS imaging (Fig. 1d) suggesting reduced collagen deposition. When mice were sacrificed at the end of the experiment, we were able to assess the egg burden of the liver tissue and also measure the collagen gene expression by qPCR. There was a significant increase in collagen mRNA expression in the $\mathrm{SjC}$ infected mice compared with control animals (Fig. 1e, $p<0.001)$. PZQ treatment resulted in a marked decrease in collagen gene expression compared with untreated SjC-infected mice $(p \leq 0.01)$. No statistical difference in collagen mRNA expression was observed in the PZQ-treated mice compared with the uninfected controls $(p>0.05)$. Furthermore, PZQ treatment resulted in a reduction in liver egg numbers (EPG) (Fig. 1f); while not reaching statistical significance, this trend showed the effects of parasite clearance on reduced egg deposition in the liver, which was associated with both decreased visualised collagen deposition and decreased collagen mRNA expression due to PZQ treatment 

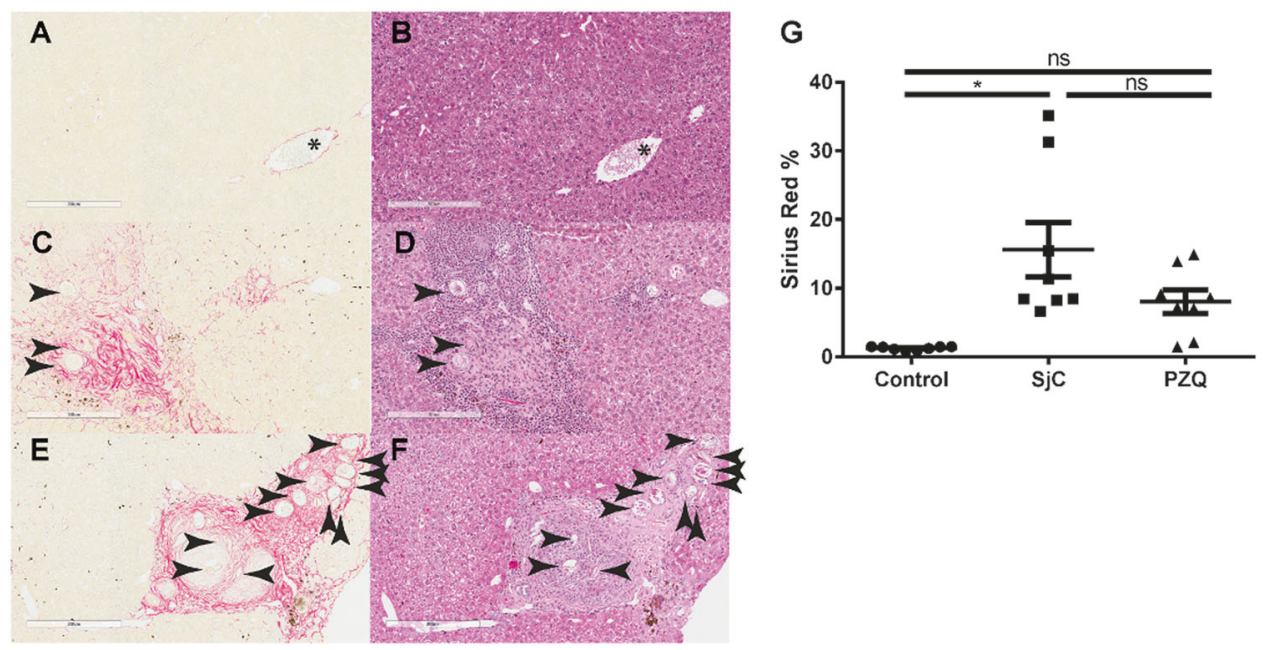

Fig. 2 Histological analysis of collagen deposition after $\mathrm{SjC}$ infection. a, c, e Picro-Sirius Red staining for collagen fibres; b, d, e haematoxylin and eosin (H\&E) staining for general cellular features; $\mathbf{a}, \mathbf{b}$ control mice; c, d SjC-infected animals; e, f SjC- infected and PZQtreated animals. Asterisk indicates blood vessel. Arrow heads indicate schistosome eggs. Scale bar represents $300 \mu \mathrm{m}$. g Analysis of Aperio quantification of Sirius Red staining ( $n=8$ animals treatment group). Data are presented as mean \pm SEM. Statistics calculated using oneway ANOVA with Tukey post-test. * $p \leq 0.05$

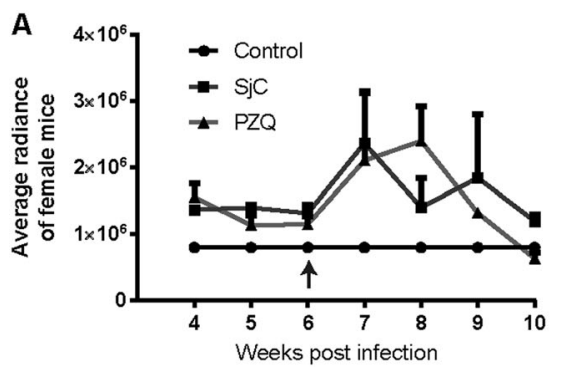

Fig. 3 Collagen bioluminescence during $\mathrm{SjC}$ infection segregated by host animal sex. a Female mice and average radiance of procollagen $\alpha 2$ (I) luciferase bioluminescence measured weekly over the infection time course $(n=5)$. Statistics calculated using two-way ANOVA with Tukey post-test: Week 4 (**Control vs SjC), Week 5 (**Control vs SjC), Weeks 7, 8, 9 (*Control vs PZQ). b Male mice and average radiance of procollagen $\alpha 2$ (I) luciferase bioluminescence measured

(Fig. 1b-d and e, respectively). These data support the value of IVIS imaging as a tool to follow collagen deposition in real time and hepatic fibrosis regression in SjC-infected and PZQ-treated Coll Luc + mice.

\section{Sirius Red staining at time course endpoint}

Collagen deposition and hepatic fibrosis have traditionally been visualised using Picro-Sirius Red histochemical staining of liver tissue sections. Liver tissue from mice was collected at the completion of the imaging study and subjected to Picro-Sirius Red histochemistry. Uninfected control mice exhibited minimal Sirius Red staining (Fig. 2a)

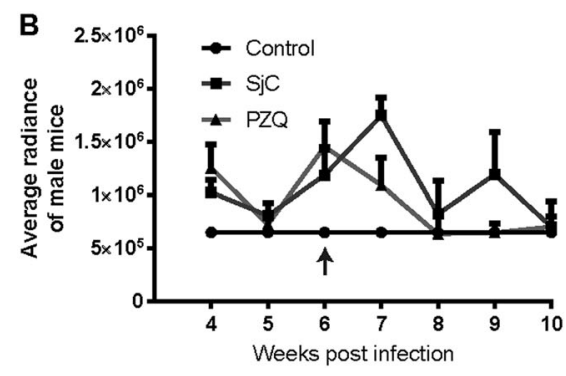

weekly over the infection time course $(n=5)$. Statistics calculated using two-way ANOVA with Tukey post-test: Week 4 (*Control vs PZQ), Week 6 (*Control vs $\mathrm{SjC}$, ***Control vs PZQ), Week 7 (****Control vs $\mathrm{SjC},{ }^{*} \mathrm{SjC}$ vs PZQ), Week 9 (*Control vs $\left.\mathrm{SjC}\right)$. Arrows represent onset of PZQ treatment. $* p \leq 0.05$, $* * \leq 0.01$, $* * * p \leq 0.001, * * * * p \leq 0.0001$

with no observed infiltration of immune cells (Fig. 2b). In contrast, mice that had been infected with $S$. japonicum (SjC) showed heavy deposition of collagen around deposited eggs (Fig. 2c), with H\&E staining revealing immune cell infiltrate surrounding deposited eggs, characteristic of granuloma (Fig. 2d). PZQ-treated mice had similar levels of collagen to mice with active infections. This was apparent when the entire histological section was quantitated (Fig. 2g); however, regions of historical collagen deposition (Fig. 2e) and infiltrated immune cells (Fig. 2f) were still evident around dead schistosome eggs. In order to quantitate Picro-Sirius Red staining across the entire region, stained tissue sections were scanned using an Aperio slide scanner to permit digital analysis and the ImageScope 
A

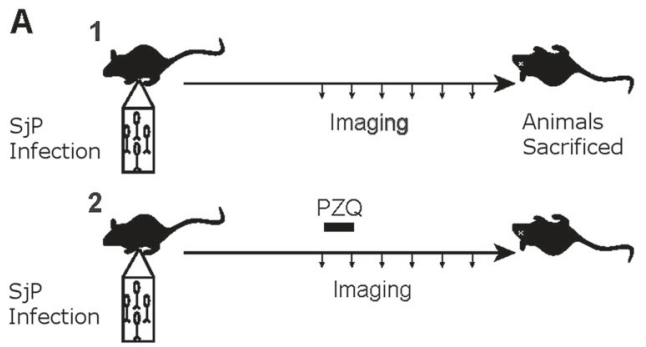

3

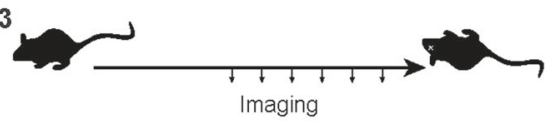

D

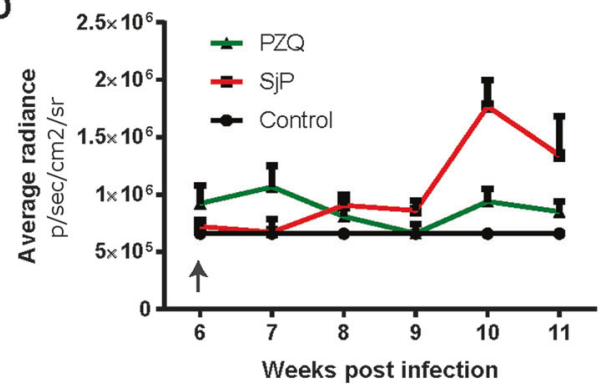

E

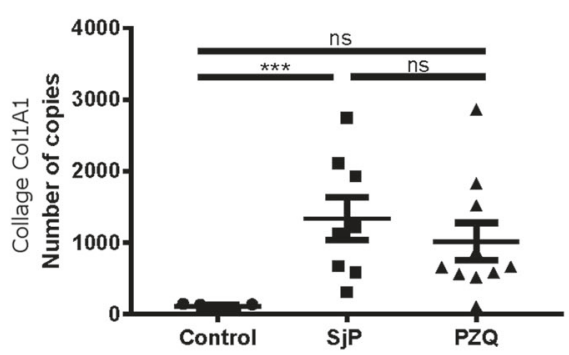

Fig. 4 Collagen deposition during $\mathrm{SjP}$ infection measured by IVIS dynamic imaging. a Experimental overview: Mice were separated into three cohorts: Group 1 was infected with $35 \mathrm{SjC}$ cercariae and imaged weekly from weeks 6 to 11; Group 2 was infected with $35 \mathrm{SjC}$ cercariae, imaged weekly from weeks 6 to 11 and treated with PZQ between weeks 6 and 7 to clear infection; Group 3 was an uninfected control group, imaged weekly from weeks 6 to 11 of the experiment. Mice were sacrificed after week 11. Representative images of $\mathbf{b}$ control and c SjP-infected mice undergoing IVIS imaging. Radiance scale (range) in $\mathrm{p} / \mathrm{s} / \mathrm{cm}^{2} / \mathrm{sr}$. d Average radiance of procollagen $\alpha 2$ (I) luciferase bioluminescence measured weekly over the infection time

software was used to quantify collagen deposition as we previously reported $[8,9,11]$. S. japonicum infection induced a significant increase in collagen deposition (Fig. 2g) compared with the controls. It is important to note that Picro-Sirius Red histochemistry differs from IVIS imaging as it detects total collagen fibres present in the tissue, while IVIS imaging reflects newly translated/ deposited collagen rather than existing collagen. It is also important to consider that histology will reflect both recent and earlier events in the tissue, and the lack of statistical differences between infected and PZQ-treated mice might have reflected this situation.
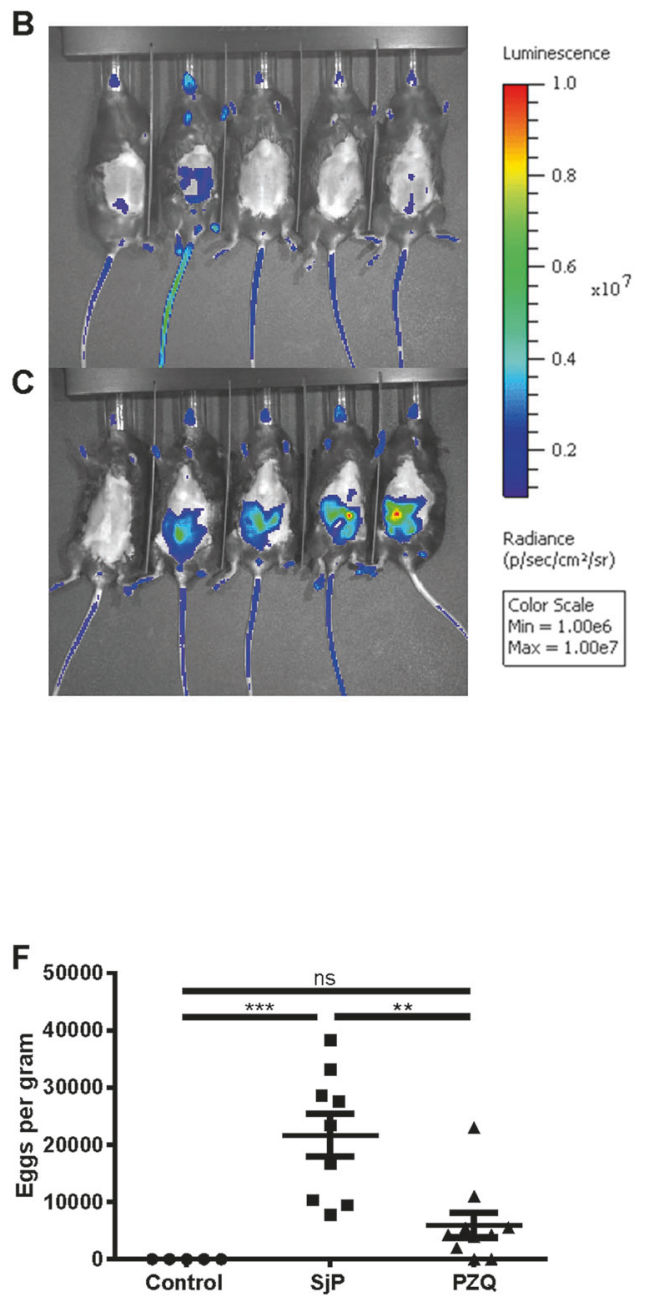

course. Arrow represents onset of PZQ treatment. Statistics calculated using two-way ANOVA with Tukey post-test: Week $10(* * * *$ Control vs S.jP, ***SjP vs PZQ), Week 11 (*Control vs SjP). e Real-time PCR of collagen gene expression in mice from the infected, PZQ-treated and control groups. Data are presented as means \pm SEM. Statistics calculated using one-way ANOVA with Tukey post-test. f Enumeration of eggs deposited in the liver during $\mathrm{SjC}$ infection. Data are presented as means \pm SEM. Statistics calculated using two-way (d) or one-way ANOVA $(\mathbf{e}, \mathbf{f})$ with Tukey post-test. $* p \leq 0.05, * * p \leq 0.01$, $* * * p \leq 0.001, * * * * p \leq 0.0001$

\section{Animal model gender differences in Coll Luc + mouse imaging}

During the initial $\mathrm{SjC}$ experiment, both male and female mice were used as they are both considered permissive hosts for schistosome infections. When these data were re-analysed and stratified by gender, we identified differences in the imaging outcomes (Fig. 3). Female mice displayed a stronger bioluminescence average radiance but showed a delayed (week 7) response after PZQ treatment (Fig. 3a). In contrast, male mice tended to have lower overall detectable bioluminescence under all 

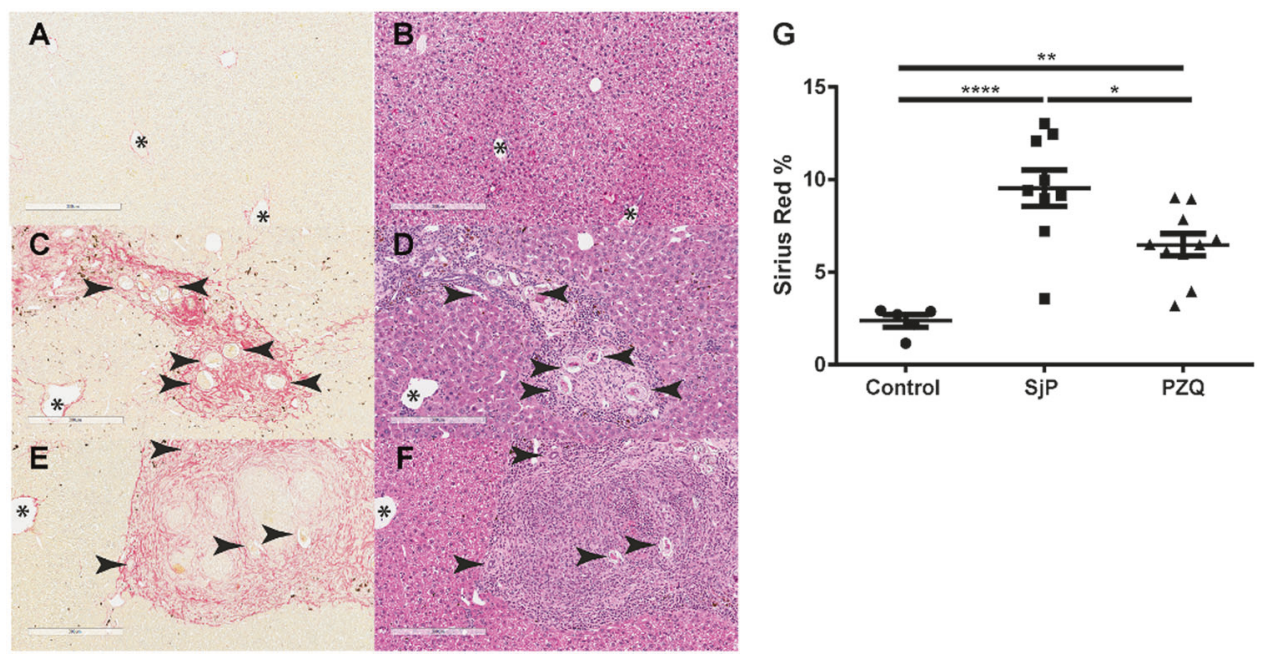

Fig. 5 Histological analysis of collagen deposition after $\mathrm{SjP}$ infection. $\mathbf{a}, \mathbf{c}$, e Picro-Sirius Red staining for collagen fibres; $\mathbf{b}$, $\mathbf{d}, \mathbf{f}$ haematoxylin and eosin (H\&E) staining for general cellular features; a, b control mice; c, d SjP-infected animals; e, f SjP-infected and PZQtreated animals. Asterisk indicates blood vessel. Arrow heads indicate schistosome eggs. Scale bar represents $300 \mu \mathrm{M}$. g Analysis of Aperio quantification of Sirius Red staining ( $n=\mathrm{SjP} 14$, PZQ 10, Control 5 animals). Data are presented as mean \pm SEM. Statistics calculated using one-way ANOVA with Tukey post-test. *p $\leq 0.05, * * p \leq 0.01$, $* * * * p \leq 0.0001$ experimental conditions, whereas the effects of $\mathrm{PZQ}$ treatment were observable rapidly after treatment, from week 6 post-challenge infection (Fig. 3b).

\section{SjP-infected Coll Luc + mice}

\section{Collagen deposition visualised using IVIS imaging and qPCR analysis}

To complement, contrast and improve on our findings gained from the $\mathrm{SjC}$ model of infection, we performed a female only mouse time course using $\mathrm{SjP}$ parasites (Fig. 4a). Being less pathogenic, we were able to infect mice with more $\mathrm{SjP}$ cercariae (35 cercariae compared with $14 \mathrm{SjC}$ cercariae). This ensured we were able to follow the infection over time and also gave a more consistent rate of egg deposition due to a higher likelihood of male-female worm pairs. Mice not infected were assessed by liver histology and egg counts and acted as controls.

As observed with the $\mathrm{SjC}$ infection (Fig. 1b-c), differences in bioluminescence were evident between control mice (Fig. 4b) and SjP-infected mice (Fig. 4c) imaged after luciferin injection. Variations in control mice signal intensities were observed, but all signals were normalised to the all-control animals. Some parasite-challenged mice were shown not to be infected following sacrifice (see first mouse on left of Fig. 4c) and these individuals were removed from the infected group analyses. After data collection, normalisation and graphing of the bioluminescence data (Fig. 4d), we were able to differentiate between infected and control mice ( $p=0.0007$, one-way ANOVA) and were also able to observe a decline in the deposition of new collagen following PZQ treatment ( $p=0.037$, two-way ANOVA). On sacrifice, a portion of mouse liver was used to assess collagen mRNA expression by qPCR (Fig. 4e). There was a significant increase in collagen mRNA expression in $\mathrm{SjP}$ mice compared with controls (Fig. 4e, $p<0.001$ ). PZQ treatment resulted in a decrease in collagen gene expression compared with untreated $\mathrm{SjP}$ mice, but this did not reach statistical significance, due to two individual outliers. SjP-infected mice had significantly more liver EPG than uninfected control mice $(p \leq 0.001)$ (Fig. 4f). Furthermore, PZQ treatment resulted in a significant decrease in egg counts compared with SJP-infected mice $(p \geq 0.01)$; however, when EPG in the PZQ-treated mice and controls were compared the difference was not statistically significant. Together these data support the utility of IVIS imaging as a method to monitor ongoing schistosomeinduced fibrosis and to assess fibrosis regression with PZQ treatment.

\section{Sirius Red staining during time course and at endpoint}

Similar to our findings with $\mathrm{SjC}$, Sirius Red staining of the livers of uninfected control mice (Fig. 5a) showed minimal collagen deposition while H\&E staining showed no lymphocyte infiltration (Fig. 5b). In contrast, SjP-infected mice showed significant collagen deposition around eggs lodged in the liver (Fig. 5c) and this correlated with a large infiltration of lymphocytes, as visualised by H\&E staining (Fig. 5d). PZQ-treated mice demonstrated 


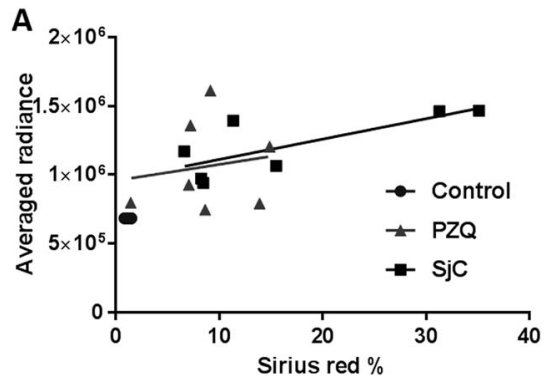

Fig. 6 Correlation of collagen bioluminescence and histological methods from schistosome-infected mice, with or without praziquantel treatment, at time course endpoint. Average radiance from procollagen

reduced levels of collagen across the tissue sections, but areas of collagen deposition (Fig. 5e) and infiltrated immune cells (Fig. 5f) were still clearly present around entrapped schistosome eggs. When Sirius Red staining was quantified using the ImageScope software, there was a significant increase in collagen deposition in the $\mathrm{SjP}$ infected mice compared with uninfected controls $(p \leq$ 0.0001); PZQ treatment resulted in a marked decrease in collagen when compared with untreated $\mathrm{SjP}$ mice (Fig. 5g; $p \leq 0.05)$. These differences reflect the differences in egg burden between the infected and recovering livers and mirror the observed changes in collagen using IVIS imaging (Fig. 4d).

\section{Correlation of IVIS imaging with Sirius Red staining or collagen gene expression during and after $\mathrm{SjC}$ and SjP infections}

To better appreciate the advantages and limitations of the whole-animal technique, we correlated the data obtained using this method with more traditional invasive collagen and quantitation approaches. Figures 6-8 present the correlation between the three techniques measuring collagen: bioluminescence, Sirius-Red histology, and real-time PCR. Data were used from both the SjC and $\mathrm{SjP}$ infections, and all time points where animals were sacrificed were used, so that the corresponding tissue could be obtained to allow histological processing and mRNA isolation. Correlations between Sirius-Red staining and bioluminescence (Fig. 6a, b) during the $\mathrm{SjC}$ infection presented correlations of $R^{2}=0.5764$ (active infection) and 0.02512 (PZQ treatment), while the SjP infection presented correlations of $R^{2}=0.762$ for active infection and 0.321 for PZQ-treated animals.

Figures 7 and 8 show additional correlations, including average radiance from luciferase bioluminescence compared with real-time PCR quantitation (COL1A1), PicroSirius Red staining compared with real-time PCR quantitation of COL1A1, average radiance compared with

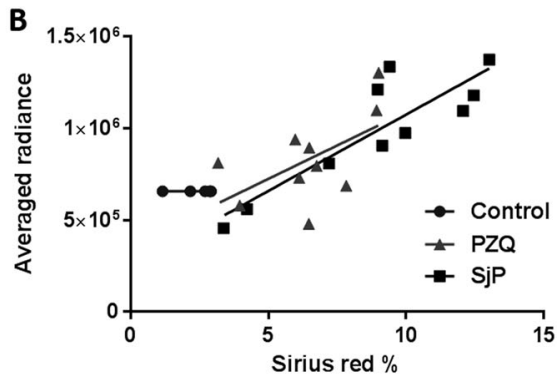

$\alpha 1$ (I) luciferase bioluminescence versus percentage of positive PicroSirius Red staining for collagen fibres. a SjC infection, $R^{2}=0.576$ (SjC), 0.025 (PZQ); b SjP infection, $R^{2}=0.762(\mathrm{SjP}), 0.321$ (PZQ)

parasitological burden (EPG), and Picro-Sirius Red staining compared with parasitological burden. The strongest correlations were evident from the $\mathrm{SjC}$ time course during the active infection, on comparing bioluminescence and egg burden (Fig. $7 \mathrm{c} ; R^{2}=0.353$ ) and Sirius-red staining and egg burden (Fig. 7d; $R^{2}=0.201$ ). Additional strong correlations were evident in PZQ-treated animals infected with $\mathrm{SjC}$ when compared with histological staining and egg burden (Fig. $7 \mathrm{~d} ; R^{2}=0.572$ ). With the SjP-infected animals, the strongest correlations were evident between the bioluminescence and egg burden in active infection $\left(R^{2}=0.469\right)$ and in PZQ treated animals $\left(R^{2}=0.673\right.$; Fig. $\left.8 \mathrm{c}\right)$.

\section{Discussion}

Human schistosomiasis results in considerable chronic disease and morbidity in many regions of the world [13], with pathology primarily arising from the tissue deposition of schistosome eggs and the formation of collagenous granuloma [4]. Traditionally, histology or qPCR have been used to measure the degree of fibrosis in schistosomiasis and other liver diseases [14, 15], although these methods have limitations as will be discussed further below. The value of in vivo approaches to understand the biology and lifecycle of schistosome parasites has been recently reviewed [16]. We sought to demonstrate the capacity of whole-animal imaging in characterising the chronic pathology caused by the eggs of schistosome blood flukes. Histological approaches for characterising hepatic schistosomiasis progression can provide high-quality resolution in terms of cellular populations and the presence of key extracellular (matrix) structures, such as collagen [17]. However, the major limitation of these methods is the static, cross-sectional or one off "snap-shot" that they provide. Here we have explored the capacity for bioluminescent whole-animal imaging to monitor the dynamic nature of liver fibrosis development induced as a result of experimental hepatic schistosomiasis. We demonstrate the utility 

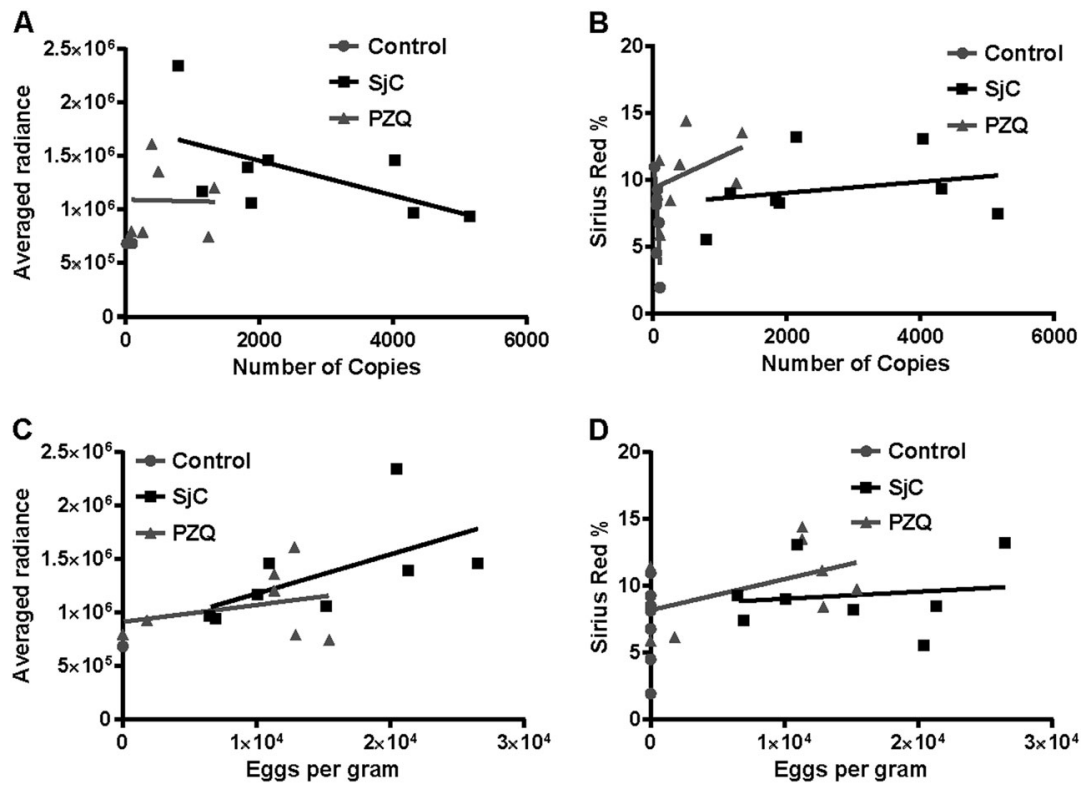

Fig. 7 Correlation of collagen quantitation methods for SjC-infected mice, with or without praziquantel treatment, at time course endpoint, with parasitological burden. a Average radiance from procollagen $\alpha 1$ (I) luciferase bioluminescence versus real-time PCR quantitation of COL1A1, $R^{2}=0.015(\mathrm{SjC}), 0.053(\mathrm{PZQ})$; (b) percentage of positive Picro-Sirius Red staining for collagen fibres versus real-time PCR quantitation of COL1A1, $R^{2}=0.009(\mathrm{SjC}), 0.355(\mathrm{PZQ})$; c average
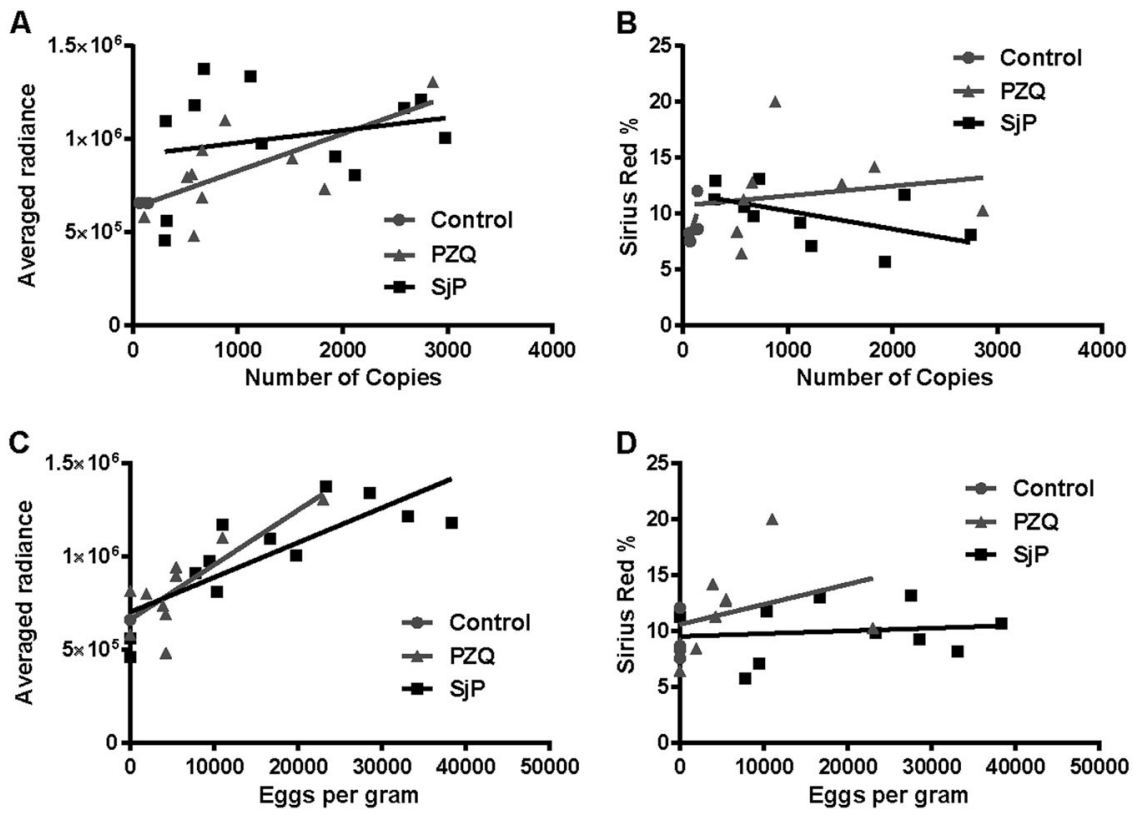

Fig. 8 Correlation of collagen quantitation methods for $\mathrm{SjP}$-infected mice, with or without praziquantel treatment, at time course endpoint, with parasitological burden. a Average radiance from procollagen $\alpha 1$ (I) luciferase bioluminescence versus real-time PCR quantitation of COL1A1, $R^{2}=0.144(\mathrm{SjP}), 0.454(\mathrm{PZQ})$; b percentage of positive Picro-Sirius Red staining for collagen fibres versus real-time PCR quantitation of COL1A1, $R^{2}=0.295(\mathrm{SjP}), 0.035(\mathrm{PZQ})$; c average

radiance from procollagen $\alpha 1$ (I) luciferase bioluminescence versus parasitological burden expressed as eggs per gram of liver, $R^{2}=0.353$ ( $\mathrm{SjC}$ ), 0.079 (PZQ); d percentage of positive Picro-Sirius Red staining for collagen fibres versus parasitological burden expressed as eggs per gram of liver, $R^{2}=0.201(\mathrm{SjC}), 0.572(\mathrm{PZQ})$. Control (circle), infected $\mathrm{SjC}$ (square) and infected then PZQ treated (triangle)

radiance from procollagen $\alpha 1$ (I) luciferase bioluminescence versus parasitological burden expressed as eggs per gram of liver, $R^{2}=0.469$ (SjP), 0.673 (PZQ); d percentage of positive Picro-Sirius Red staining for collagen fibres versus parasitological burden expressed as eggs per gram of liver, $R^{2}=0.016(\mathrm{SjP}), 0.105$ (PZQ). Control (circle), infected $\mathrm{SjP}$ (square) and infected then PZQ treated (triangle) 
of transgenic collagen luciferase reporter mice (B6.Coll $1 \mathrm{~A}-\mathrm{luc}^{+}$) as an effective model for measuring disease progression resulting from collagen deposition, both during time courses of infection and treatment-induced recovery. We found that collagen transcription and deposition, which coincides with schistosome egg laying, was detectable using this method in B6.Coll 1A-luc ${ }^{+}$mice infected with two distinct geographical strains $(\mathrm{SjC}$ and $\mathrm{SjP})$ of $S$. japonicum, which exhibit differences in infectivity, patency and fecundity. Additionally, we sought to compare bioluminescence with traditional histological analysis of collagen deposition using Picro-Sirius Red staining and realtime PCR. We found that bioluminescent imaging provided a dynamic temporal measure of ongoing collagen gene transcription. While the deposition of collagen during infection is useful, the capacity to visualise changes in collagen deposition after the infection was cleared and, during recovery or fibrosis regression, provided a unique insight into this phase of the disease.

As another indicator of liver damage and fibrosis, myofibroblasts have been visualised in a whole-animal approach using fluorescently labelled antibodies [18]. In this study, the $\mathrm{CCl}_{4}$ model of hepatic damage could be clearly shown to correlate with increased hepatic myofibroblast numbers, with observation validated by tradition histology. Furthermore, while this study primarily compared treated and control mice, this approach is complementary to our technical approach and mouse model. The visualisation of myofibroblast recruitment to an organ complements our imaging of collagen transcription and would strengthen the disease time course and the recovery phases we characterised.

While whole-animal imaging is a technique that has not been widely applied to parasite-driven liver damage and, specifically, fibrosis, some reports are available. For example, bioluminescent trypanosomes have been successfully imaged in live mice to track parasite migration to specific host organs during the establishment of disease [19]. A similar approach could be undertaken in the future on schistosomes, but the application of transgenic methods for studying blood flukes is still in its infancy [20]. Whereas bioluminescence schistosomes are not yet available, other in vivo methods have been used to image parasites within animal models. The voracious uptake of glucose by adult schistosomes [10] and their unique gut enzyme profile provided the opportunity to label parasites in vivo and image using carbohydrate radiotracers and positron emission tomography [21], and enzyme-activated imaging compounds and fluorescence molecular tomography [22]. Both of these methods were able to identify schistosomes adjacent to host liver demonstrating the link between the parasite and the known site of pathology. Imaging schistosome-induced pathology and the impact on organ function has been reported in hepatic schistosomiasis using radiolabelled compounds [23]. Through the labelling of a surface receptor (Asialoglycoprotein receptor), found only on healthy hepatocytes, a significant decrease in labelling $(p<0.01)$ and liver health was observed during the progression of a $S$. mansoni infection in mice, with the positive correlation of this output with histology and serum biochemical markers, similar to that presented here, provided validation of these imaging methods.

Schistosome-induced granulomas comprise multiple cellular populations [4]. The measurement of additional cellular markers using whole-animal imaging would promote further analysis of the dynamics of hepatic diseases, such as schistosomiasis, in terms of both disease progression and recovery. For example, neutrophils have been tracked in an experimental colitis model [24], and the potential for imaging macrophages in a mouse model has also been investigated [25]. Other prospective avenues utilising whole-animal imaging for fibrotic hepatic disease models include investigating components of the extracellular matrix such as remodelling enzymes including matrix metalloproteinases, which can be resolved by the use of nanoprobes or bioluminescent substrates [26]. Vascular endothelial growth factor (VEGF), recently reported as being of importance in chronic schistosomiasis [27], is another marker that could be further evaluated by wholeanimal imaging. Transgenic mouse models are available that can report the presence of VEGF as a VEGF-green fluorescent protein [28] or transcription of VEGF receptor 2 through a luciferase reporter [29]. The pro-fibrotic activity of VEGF in these animal models could be used to investigate the potential interaction between this growth factor and hepatic stellate cells (HSCs) during schistosomiasis. HSCs play a pivotal role in the formation of schistosome egg-induced hepatic granuloma $[4,30]$ through the deposition of collagen, the extracellular matrix component imaged in the current study.

An important distinction between bioluminescent imaging used here and more traditional histological methods is that the former measures the production of new collagen as it is being transcribed, whereas the latter measures the total amount of histological collagen present, regardless of when it is deposited in tissues [14]. As a result, bioluminescent imaging is able to monitor dynamic changes in collagen transcription and translation over time, allowing both the pinpointing of collagen deposition in response to trapped hepatic eggs and also responses to treatment and clearance of an active infection. Observing the real-time dynamics of collagen deposition provides a powerful tool for determining the speed and efficacy of different treatments in realtime infections. The ability to follow collagen deposition as a proxy for fibrosis also allows for the study of animals 
developing fibrosis before treatment is administered, thereby providing a more clinically relevant experimental setting.

Another significant benefit of using dynamic bioluminescent imaging rather than traditional methods is that significantly fewer animals are required, as each animal can be assessed over the duration of the treatment time course without the need for their sacrifice at different treatment intervals. The principles of the 3Rs (Replacement, Reduction and Refinement) [31] is a research priority for many funding bodies, including the UK - Medical Research Council (MRC) and NC3Rs. For example, to follow a schistosome (SjC) infection, as we have done here using only traditional histology at each time point, would require three groups of five mice at each time point (i.e. SjC, PZQ and Control). With time points required at weeks 4-10 post-infection (i.e. seven time points, a total of 105 mice would normally be required. Using our alternative dynamic bioluminescent imaging method in the $\mathrm{SjC}$ experiment, we used 5 control mice, $12 \mathrm{SjC}$-infected and 10 PZQ-treated mice for a total of 27. Furthermore, by following the same animals over time it is possible to avoid artefacts of biological variability where mice with different individual infection characteristics are sacrificed and compared at different time points. We believe that bioluminescence imaging as a model of hepatic schistosomiasis will reduce the number of mice needed per experiment, while increasing the quality of the experimental data that is generated.

Despite providing a number of advantages, bioluminescent imaging also has some limitations. For example, we observed differences in the sensitivity of the average radiance measurements between infected male and female mice. In SjC infection and presented in Fig. 3, there was a reduction in signal by week 10 that was more pronounced in male animals. We found it preferable to use female mice for the imaging experiments due to the lower signal obtained with males. This may have been due to the latter having a thicker, tougher skin [32], which may have inhibited the average radiance signal. Additionally, male mice are more prone to fighting [33] with the resulting wound repair (scar) likely involving collagen deposition, a feature that potentially could complicate interpretation of results and increase background luminescence. Accordingly, we recommend future studies be performed using female mice for improved imaging signal-to-noise ratios. Variations in weekly radiance was also noted and could reflect variation in egg production by parasites or impact of host responses on the parasite. The addition of larger mouse group sizes would help to minimalise this variation. Additionally in the $\mathrm{SjC}$ infections where resulting pathology was higher, mice needed to be culled earlier for ethical considerations, and this may influence the weekly signal averages obtained. The use of low pathology $\mathrm{SjP}$ parasites reduced this potential source of variation and experimental design limitation.

Bioluminescent imaging correlated with other measures of infection and fibrosis that were assessed, such as the central driver of infection, the number of eggs present in the liver and also qPCR for collagen mRNA. There was a strong correlation when bioluminescence was compared with Sirius Red histochemical analysis for both the $\mathrm{SjP}$ - and SjC-infected mice. Correlations between Sirius Red staining and bioluminescence were, however, less evident in the recovering livers post-PZQ treatment. This latter observation likely reflects Sirius Red measurement of total tissue collagen, whereas bioluminescence measures new collagen at the time of imaging. Overall, these results support bioluminescent imaging as a more sensitive and accurate technique for monitoring changes in collagen deposition in real time in individual animals using this infection model, with the added advantage that considerably reduced animal numbers are required.

\section{Conclusions}

This whole-animal collagen luciferase model we present has effectively reinforced the concept of the dynamic nature of the process of hepatic schistosomiasis first advanced by Pearce and MacDonald [34], with the commencement of egg laying and the induction of a $\mathrm{T}$ helper type 2 immune response being mirrored in our findings through the marker of collagen bioluminescence. Furthermore, the peaking, plateauing and reduction of the fibrotic response during the development of chronic hepatic schistosomiasis is also apparent using this model so that many of the features associated with the disease may be better studied using a whole-animal imaging approach of the type described here.

The various advantages of using whole-animal imaging for fibrosis is a distinct addition to the tool box of techniques available to studying hepatic disease caused by schistosomes. The future testing of antischistosomal and/or antifibrotic compounds would benefit from the dynamic data that is obtainable using these methods. Similarly, the additional parameter of collagen deposition would add value to the evaluation of potential vaccines assessed in mice, in targeting the parasite and the pathology they cause. The real-time quantitation of the establishment and regression of collagen deposition in clearly defined organs such as the liver will make the prioritisation and higher throughput evaluation of these therapeutics more feasible in future.

Acknowledgements The technical support of Mary Duke (QIMRB) is much appreciated. The technical service of the Histology department QIMR Berghofer is acknowledged. This research was supported by 
Program (APP1132975) and Project (APP1098244) grants from the National Health and Medical Research Council (NHMR) of Australia. DPM is an NHMRC Senior Principal Research Fellow (APP1102926) and Senior Scientist at QIMR Berghofer.

\section{Compliance with ethical standards}

Conflict of interest The authors declare that they have no conflict of interest.

Ethics statement The conduct and procedures involving animal experiments was performed with the approval of the QIMR Berghofer Medical Research Institute Animal Ethics Committee (project number A9912-00-043). If necessary, mice were culled if they displayed excessive discomfort.

\section{References}

1. Colley DG, Bustinduy AL, Secor WE, King CH. Human schistosomiasis. Lancet. 2014;383:2253-64.

2. Hope M, Duke M, McManus DP. A biological and immunological comparison of Chinese and Philippine Schistosoma japonicum. Int J Parasitol. 1996;26:325-32.

3. Gobert GN, You H, Jones MK, McInnes R, McManus DP. Differences in genomic architecture between two distinct geographical strains of the blood fluke Schistosoma japonicum reveal potential phenotype basis. Mol Cell Probes. 2013;27:19-27.

4. Burke ML, Jones MK, Gobert GN, Li YS, Ellis MK, McManus DP. Immunopathogenesis of human schistosomiasis. Parasite Immunol. 2009;31:163-76.

5. Wynn TA, Thompson RW, Cheever AW, Mentink-Kane MM. Immunopathogenesis of schistosomiasis. Immunol Rev. 2004; 201:156-67.

6. Bou-Gharios G, Garrett LA, Rossert J, Niederreither K, Eberspaecher H, Smith C, et al. A potent far-upstream enhancer in the mouse pro alpha 2(I) collagen gene regulates expression of reporter genes in transgenic mice. J Cell Biol. 1996;134: 1333-44.

7. Melino M, Gadd VL, Alexander KA, Beattie L, Lineburg KE, Martinez M, et al. Spatiotemporal characterization of the cellular and molecular contributors to liver fibrosis in a murine hepatotoxic-injury model. Am J Pathol. 2016;186:524-38.

8. Chuah C, Jones MK, McManus DP, Nawaratna SK, Burke ML, Owen HC, et al. Characterising granuloma regression and liver recovery in a murine model of schistosomiasis japonica. Int $\mathbf{J}$ Parasitol. 2016;46:239-52.

9. Perry CR, Burke ML, Stenzel DJ, McManus DP, Ramm GA, Gobert GN. Differential expression of chemokine and matrix re-modelling genes is associated with contrasting schistosomeinduced hepatopathology in murine models. PLoS Negl Trop Dis. 2011;5:e1178.

10. You H, Gobert GN, Duke MG, Zhang W, Li Y, Jones MK, et al. The insulin receptor is a transmission blocking veterinary vaccine target for zoonotic Schistosoma japonicum. Int J Parasitol. 2012; 42:801-7.

11. Burke ML, McManus DP, Ramm GA, Duke M, Li Y, Jones MK, et al. Temporal expression of chemokines dictates the hepatic inflammatory infiltrate in a murine model of schistosomiasis. PLoS Negl Trop Dis. 2010;4:e598.

12. Hoffmann KF, Johnston DA, Dunne DW. Identification of Schistosoma mansoni gender-associated gene transcripts by cDNA microarray profiling. Genome Biol. 2002;3: RESEARCH0041.
13. Murray CJ, Vos T, Lozano R, Naghavi M, Flaxman AD, Michaud C, et al. Disability-adjusted life years (DALYs) for 291 diseases and injuries in 21 regions, 1990-2010: a systematic analysis. Lancet. 2013;380:2197-223.

14. Lattouf R, Younes R, Lutomski D, Naaman N, Godeau G, Senni $\mathrm{K}$, et al. Picrosirius red staining: a useful tool to appraise collagen networks in normal and pathological tissues. J Histochem Cytochem. 2014;62:751-8.

15. Wang L, Potter JJ, Rennie-Tankersley L, Novitskiy G, Sipes J, Mezey E. Effects of retinoic acid on the development of liver fibrosis produced by carbon tetrachloride in mice. Biochim Biophys Acta. 2007;1772:66-71.

16. Skelly PJ. The use of imaging to detect schistosomes and diagnose schistosomiasis. Parasite Immunol. 2013;35:295-301.

17. Amaral KB, Silva TP, Dias FF, Malta KK, Rosa FM, CostaNeto SF, et al. Histological assessment of granulomas in natural and experimental Schistosoma mansoni infections using whole slide imaging. PLoS ONE. 2017;12:e0184696.

18. Luli S, Di Paolo D, Perri P, Brignole C, Hill SJ, Brown H, et al. A new fluorescence-based optical imaging method to noninvasively monitor hepatic myofibroblasts in vivo. J Hepatol. 2016;65:75-83.

19. Henriques C, Henriques-Pons A, Meuser-Batista M, Ribeiro AS, de Souza W. In vivo imaging of mice infected with bioluminescent Trypanosoma cruzi unveils novel sites of infection. Parasit Vectors. 2014;7:89.

20. Yan HB, Smout MJ, Ju C, Folley AE, Skinner DE, Mann VH, et al. Developmental sensitivity in Schistosoma mansoni to puromycin to establish drug selection of transgenic schistosomes. Antimicrob Agents Chemother. 2018;62:e02568-17.

21. Salem N, Balkman JD, Wang J, Wilson DL, Lee Z, King CL, et al. In vivo imaging of schistosomes to assess disease burden using positron emission tomography (PET). PLoS Negl Trop Dis. 2010;4:e827.

22. Krautz-Peterson G, Ndegwa D, Vasquez K, Korideck H, Zhang J, Peterson JD, et al. Imaging schistosomes in vivo. FASEB J. 2009;23:2673-80.

23. Cheng PC, Chiang PF, Lee KM, Yeh CH, Hsu KL, Liu SW, et al. Evaluating the potential of a new isotope-labelled glyco-ligand for estimating the remnant liver function of schistosoma-infected mice. Parasite Immunol. 2013;35:129-39.

24. Murphy CT, Moloney G, Hall LJ, Quinlan A, Faivre E, Casey P, et al. Use of bioluminescence imaging to track neutrophil migration and its inhibition in experimental colitis. Clin Exp Immunol. 2010;162:188-96.

25. Pajarinen J, Lin TH, Sato T, Loi F, Yao Z, Konttinen YT, et al. Establishment of green fluorescent protein and firefly luciferase expressing mouse primary macrophages for in vivo bioluminescence imaging. PLoS ONE. 2015;10:e0142736.

26. Lee H, Kim YP. Fluorescent and bioluminescent nanoprobes for in vitro and in vivo detection of matrix metalloproteinase activity. BMB Rep. 2015;48:313-8.

27. Luo J, Liang Y, Kong F, Qiu J, Liu X, Chen A, et al. Vascular endothelial growth factor promotes the activation of hepatic stellate cells in chronic schistosomiasis. Immunol Cell Biol. 2017; 95:399-407.

28. Fukumura D, Xavier R, Sugiura T, Chen Y, Park EC, Lu N, et al. Tumor induction of VEGF promoter activity in stromal cells. Cell. 1998;94:715-25.

29. Zhang N, Fang Z, Contag PR, Purchio AF, West DB. Tracking angiogenesis induced by skin wounding and contact hypersensitivity using a Vegfr2-luciferase transgenic mouse. Blood. 2004; 103:617-26.

30. Carson JP, Ramm GA, Robinson MW, McManus DP, Gobert GN. Schistosome-induced fibrotic disease: the role of hepatic stellate 
cells. Trends Parasitol. 2018;34:524-40. https://doi.org/10.1016/j. pt.2018.1002.1005

31. MacArthur Clark J. The $3 R$ s in research: a contemporary approach to replacement, reduction and refinement. Br J Nutr. 2017;120 (s1):S1-S7.

32. Azzi L, El-Alfy M, Martel C, Labrie F. Gender differences in mouse skin morphology and specific effects of sex steroids and dehydroepiandrosterone. J Invest Dermatol. 2005;124:22-7.
33. Meakin LB, Sugiyama T, Galea GL, Browne WJ, Lanyon LE, Price JS. Male mice housed in groups engage in frequent fighting and show a lower response to additional bone loading than females or individually housed males that do not fight. Bone. 2013;54:113-7.

34. Pearce EJ, MacDonald AS. The immunobiology of schistosomiasis. Nat Rev Immunol. 2002;2:499-511. 